International Journal of Physical Research, $8(2)(2020) 40-44$
International Journal of Physical Research
SPC
Website: www.sciencepubco.com/index.php/IJPR
Research paper

\title{
Dark soliton solutions to $(2+1)$-dimensional Kundu-Mukherjee-Naskar equation via the first integral method
}

\author{
Salam Subhaschandra Singh * \\ Department of Physics, Imphal College, Imphal, 795001, Manipur, India \\ *Corresponding authorE-mail: subhasic@yahoo.co.in
}

\begin{abstract}
In the present work, the First Integral Method is being applied in finding a non-soliton as well as a soliton solution of the $(2+1)$ dimensional Kundu-Mukherjee-Naskar (KMN) equation which is a variant of the well-known Nonlinear Schrodinger ( NLS ) equation. Using the method, a dark optical soliton solution and a periodic trigonometric solution to the KMN equation have been suggested and the relevant conditions which guarantee the existence of such solutions are also indicated therein.
\end{abstract}

Keywords: Kundu-Mukherjee-Naskar Equation; First Integral Method.

\section{Introduction}

Optical solitons are pulses which compose the basic fabric of signal transmission across trans-continental and trans-oceanic distances in telecommunication engineering. In the present decade, the study of the dynamics of optical soliton propagation through optical fibers yields many promising results in the research of optical communication systems. Several equations / models have been proposed so far in the past a few decades to describe such physical phenomena and the Kundu-Mukherjee-Naskar (KMN ) equation [1- 6] is one of them. This equation was first proposed in the year 2014 by three Indian physicists namely Anjan Kundu, Abhik Mukherjee and Tapan Naskar for modelling the dynamics of two-dimensional rogue waves in ocean water and also two-dimensional ion-acoustic waves in magnetized plasmas $[7,8]$. We can consider this equation as an extension of the well-known Nonlinear Schrodinger (NLS) equation. It can also be used in describing propagation of optical wave through coherently excited resonant wave-guides in particular in the theory of bending of light beams. Various methods have been proposed so far by various authors for solving Nonlinear Evolution Equations (NLEEs) and the First Integral Method [ 9 - 16] is picked up here to find a soliton solution of KMN equation.

\section{Kundu-Mukherjee-Naskar $(\mathrm{KMN})$ equation in $(2+1)$ dimensions}

The KMN equation is written in a dimensionless form as

$i q_{t}+a q_{x y}+i b q\left(q q_{x}^{*}-q^{*} q_{x}\right)=0$

where $i=\sqrt{-1}, x$ and $y$ are spatial variables, $t$ is the temporal variable, $u(x, y, t)$ is a dependent variable depending on $x, y, t$ and giving the profile of the optical soliton or nonlinear wave envelope, the asterisk denotes complex conjugation and the subscripts indicate derivatives of the dependent variable with respect to them; the first term ensures a temporal evolution, the second term with a coefficient ' $a$ ' represents a dispersion term, the final term on the left hand side with a coefficient ' $b$ ' is the nonlinear term that is different from the conventional Kerr-type or any known non-Kerr type nonlinearity arising in the celebrated Nonlinear Schrodinger ( NLS ) equation and other earlier generalized models.

Although the equation was proposed by Kundu, Mukherjee and Naskar to describe oceanic rogue waves as well as hole waves, it may also be used in describing optical waves or soliton propagation through Erbium doped coherently excited resonant wave-guides. Further, this equation can also be used in the study of the phenomenon of bending of light beams. Due to these reasons, many researchers turned to the investigation of this equation and subsequently several findings had been published elsewhere. In the following sections, we describe an algorithm of the First Integral Method and it is applied in solving the KMN equation. 


\section{Algorithm of first integral method}

The First Integral Method, based on the ring theory of commutative algebra was first proposed by Z. S. Feng and was further developed by himself. This method had been effectively applied by many authors in solving different types of NLEEs encountered in the study of science and engineering. The main steps of this method are outlined as in the following.

We suppose that we are to solve a nonlinear partial differential equation (NLPDE) that can be expressed in the form

$F\left(u, u_{x}, u_{t}, u_{x x}, u_{x t}, u_{t t}, u_{x x x} \ldots\right)=0$

where $x, t$ are independent variables, $u$ is a dependent variable depending on $x, t$; the subscripts denote the partial derivatives of the dependent variable with respect to them and $F$ is a polynomial in $u$ and its various partial derivatives.

We introduce the following travelling wave transformations

$u=u(x, t)=U(\xi), \quad \xi=x-c t$

where $\mathrm{c}$ is a constant to be determined latter. This constant $\mathrm{c}$ is generally the constant speed of the wave propagation.

Using the above transformations, Eq. (2) is reduced to a nonlinear ordinary differential equation (NLODE) of the form

$G\left(U, U^{\prime}, U^{\prime \prime}, U^{\prime \prime \prime}, \ldots\right)=0$

where

$U^{\prime}=d U / d \xi$ etc.

Next, let us put

$U(\xi)=R(\xi), \quad U^{\prime}(\xi)=\frac{d U}{d \xi}=S(\xi)$

Then, we have a plane autonomous system

$S(\xi)=R^{\prime}(\xi), \quad S^{\prime}(\xi)=H(R(\xi), S(\xi))$

where $H$ is a polynomial in $R$ and $S$.

If we can find two first integrals to this plane autonomous system under the same conditions, then analytic solutions of the system (6) can be obtained directly. However, in general, it is really difficult for us to realize this even for one first integral, because for a given plane autonomous system, there exists neither a systematic theory that can tell us how to find its first integrals nor a logical way for telling us what these first integrals are. We may apply the Division Theorem to obtain a first integral to the system (6) which reduces Eq. (4) to an integrable first order ODE. An exact solution of Eq. (2) can be obtained by solving this first order ODE. For convenience, let us recall the Division Theorem for two variables in a complex domain $C[w, z]$.

Division Theorem: For two polynomials $P_{1}(w, z)$ and ${ }_{P_{2}(w, z)}$ in a complex domain $C[w, z]$, if $P_{1}(w, z)$ is irreducible in $C[w, z]$ and if $P_{2}(w, z)$ vanishes at all zero points of $P_{1}(w, z)$; then there exists another polynomial $P_{3}(w, z)$ in $C[w, z]$ such that $P_{2}(w, z)=P_{1}(w, z) P_{3}(w, z)$. The division theorem directly follows from the Hilbert-Nullstellensatz theorem of commutative algebra.

\subsection{Reduction of KMN Equation to a NLODE}

To reduce Eq. (1) to a NLODE, we introduce the transformations

$q(x, y, t)=U(\xi) e^{-i \eta}, \quad \xi=x+y-c t, \eta=k_{1} x+k_{2} y-\omega t+\epsilon$

where $\mathrm{c}$ is the constant soliton propagation speed, $k_{1}$ and $k_{2}$ are the soliton frequencies in the $x$ and $y$ directions respectively, $\omega$ is the wave number of the soliton and $\epsilon$ is a phase constant.

Now, we have

$q_{t}=\left(-c U^{\prime}+i \omega U\right) e^{-i \eta}$,

$q_{x}=\left(U^{\prime}-i k_{1} U\right) e^{-i \eta}$,

$q_{x y}=\left(U^{\prime \prime}-i k_{1} U^{\prime}-i k_{2} U^{\prime}-k_{1} k_{2} U\right) e^{-i \eta}$.

Now, substituting the expressions for $q$, its complex conjugate $q^{*}$ and its partial derivatives $q_{t}, q_{x}$ and $q_{x y}$ into Eq. (1), we obtain the real and the imaginary parts of the resulting equation as

Real Part:

$a U^{\prime \prime}-\left(a k_{1} k_{2}+\omega\right) U-2 b k_{1} U^{3}=0$,

Imaginary Part: 
$-\left(c+a k_{1}+a k_{2}\right) U^{\prime}=0$

Or, $c=-a\left(k_{1}+k_{2}\right)$.

\section{Application of the first integral method to Solving KMN Equation}

From Eqs. (5) and (6), we re-write

$R(\xi)=U(\xi), \quad S(\xi)=R^{\prime}(\xi)=U^{\prime}(\xi)$.

From Eq. (8), we have

$S^{\prime}(\xi)=U^{\prime \prime}(\xi)=\left(k_{1} k_{2}+\frac{\omega}{a}\right) R+\left(\frac{2 b k_{1}}{a}\right) R^{3}$.

Using Eqs. (10) and (11), we obtain the following two dimensional autonomous system

$S(\xi)=R^{\prime}(\xi), \quad S^{\prime}(\xi)=\left(k_{1} k_{2}+\frac{\omega}{a}\right) R+\left(\frac{2 b k_{1}}{a}\right) R^{3}$.

In accordance with the first integral method, it is assumed that $R(\xi)$ and $S(\xi)$ are non-trivial solutions of the autonomous system (12) and that the polynomial $Q(R(\xi), S(\xi))=\sum_{j=0}^{m} \gamma_{j}(R) S^{j}(\xi)$ is an irreducible polynomial in the complex domain $C[R, S]$ such that

$Q(R(\xi), S(\xi))=\sum_{j=0}^{m} \gamma_{j}(R) S^{j}(\xi)=0$

where $\gamma_{j}(R)(j=0,1,2,3, \ldots m)$ are polynomials in $R$ with $a_{m}(R) \neq 0$.

Here, Eq. (13) is the first integral to the autonomous system (12). From Eq. (13), it is seen that the quantity $d Q / d \xi$ is a polynomial in $R$ and $S$ and it is equal to zero. By division theorem, there exists a polynomial $g(R)+h(R) S$ in the complex domain $C[R, S]$ such that

$\frac{d Q}{d \xi}=\left(\frac{\partial Q}{\partial R}\right)\left(\frac{d R}{d \xi}\right)+\left(\frac{\partial Q}{\partial S}\right)\left(\frac{d S}{d \xi}\right)=\sum_{j=0}^{m} \gamma_{j}^{\prime}(R) S^{j+1}+\sum_{j=0}^{m} j \gamma_{j}(R) S^{j-1} S^{\prime}(\xi)$

$=\{g(R)+h(R) S\} \sum_{j=0}^{m} \gamma_{j}(R) S^{j}(\xi)$.

Then, we consider different cases with different values of $m$.

Let us consider the case with $m=1$.

In this case, using Eqs. (12), (13) and (14), we write

$\gamma_{0}^{\prime}(R) S+\gamma_{1}^{\prime}(R) S^{2}+\gamma_{1}(R)\left[\left(k_{1} k_{2}+\frac{\omega}{a}\right) R(\xi)+\left(\frac{2 b k_{1}}{a}\right) R^{3}\right]$

$=g(R) \gamma_{0}(R)+\left\{h(R) \gamma_{0}(R)+g(R) \gamma_{1}(R)\right\} S+h(R) \gamma_{1}(R) S^{2}$.

Equating coefficients of $s^{j}(j=0,1$. $)$ from both sides of Eq. (15), we have

$g(R) \gamma_{0}(R)=\gamma_{1}(R)\left[\left(k_{1} k_{2}+\frac{\omega}{a}\right) R+\left(\frac{2 b k_{1}}{a}\right) R^{3}\right]$

$\gamma_{0}^{\prime}(R)=h(R) \gamma_{0}(R)+g(R) \gamma_{1}(R)$

$\gamma_{1}^{\prime}(R)=h(R) \gamma_{1}(R)$.

Since $\gamma_{j}(j=0,1)$ are assumed to be polynomials, we conclude from Eq.(18) that $h(R)=0$ and $\gamma_{1}(R)=$ constant. For simplicity, we take $\gamma_{1}(R)=1$.

From Eqs.(16) and (17), balancing of degrees of $\gamma_{0}(R)$ and $g(R)$ results in degree $\left[\gamma_{0}(R)\right]=2$, degree $[g(R)]=1$.

Let us assume that

$\gamma_{0}(R)=A_{0}+A_{1} R+A_{2} R^{2}$

where $A_{0}, A_{1}$ and $A_{2}$ are constants to be determined latter such that $A_{2} \neq 0$.

Using Eqs. (17) and (19) together with the values of $\gamma_{1}(R)$ and $h(R)$, we obtain

$g(R)=A_{1}+2 A_{2} R$.

Substituting Eqs. (19) and (20) into Eq. (17), we write

$$
\left(A_{1}+2 A_{2} R\right)\left(A_{0}+A_{1} R+A_{2} R^{2}\right)=\left(k_{1} k_{2}+\frac{\omega}{a}\right) R+\left(\frac{2 b k_{1}}{a}\right) R^{3} .
$$

Equating coefficients of $R^{j}(j=0,1,2,3)$ from both sides of Eq. (21), we obtain the following system of algebraic equations $A_{0} A_{1}=0$, 
$A_{1}^{2}+2 A_{0} A_{2}=\left(k_{1} k_{2}+\frac{\omega}{a}\right)$

$3 A_{1} A_{2}=0$,

$2 A_{2}^{2}=\frac{2 b k_{1}}{a}$.

Symbolic computation of the above system of equations using the computational software, Mathemetica 9.0, yields the results

$A_{0}= \pm \frac{a k_{1} k_{2}+\omega}{2 \sqrt{a b k_{1}}}$,

$A_{1}=0, \quad A_{2}= \pm \sqrt{\frac{b k_{1}}{a}}$.

Substituting these values of $A_{0}, A_{1}$ and $A_{2}$ into Eqs. (19) and (20), we obtain

$\gamma_{0}(R)= \pm \frac{a k_{1} k_{2}+\omega}{2 \sqrt{a b k_{1}}} \pm \sqrt{\frac{b k_{1}}{a}} R^{2}$

and

$g(R)= \pm 2 \sqrt{\frac{b k_{1}}{a}} R$.

Substituting the values of $\gamma_{0}(R)$ and $\gamma_{1}(R)$ into Eq. (13) and recalling that

$S(\xi)=R^{\prime}(\xi)=\frac{d R}{d \xi}$, we write

$R^{\prime}(\xi) \pm \frac{a k_{1} k_{2}+\omega}{2 \sqrt{a b k_{1}}} \pm \sqrt{\frac{b k_{1}}{a}} R^{2}=0$

Eq. (24) has the solutions

$R(\xi)= \pm \sqrt{\frac{a k_{1} k_{2}+\omega}{2 b k_{1}}} \tan \left\{\sqrt{\frac{a k_{1} k_{2}+\omega}{2 a}} \xi\right\}$

if $a\left(a k_{1} k_{2}+\omega\right)>0$

and

$$
R(\xi)= \pm \sqrt{-\frac{a k_{1} k_{2}+\omega}{2 b k_{1}}} \tanh \left\{\sqrt{-\frac{a k_{1} k_{2}+\omega}{2 a}} \xi\right\}
$$

if $a\left(a k_{1} k_{2}+\omega\right)<0$.

Substituting $R(\xi)=U(\xi)$ into Eq. (7), we obtain the solution of Eq. (1) in the forms

$q(x, y, t)= \pm \sqrt{\frac{a k_{1} k_{2}+\omega}{2 b k_{1}}} \tan \left\{\sqrt{\frac{a k_{1} k_{2}+\omega}{2 a}}(x+y-c t)\right\} e^{-i \eta}$

if $a\left(a k_{1} k_{2}+\omega\right)>0$

and

$q(x, y, t)= \pm \sqrt{-\frac{a k_{1} k_{2}+\omega}{2 b k_{1}}} \tanh \left\{\sqrt{-\frac{a k_{1} k_{2}+\omega}{2 a}}(x+y-c t)\right\} e^{-i \eta}$

if $a\left(a k_{1} k_{2}+\omega\right)<0$.

In both the above solutions, $k_{1} x+k_{2} y-\omega t+\epsilon$ where $k_{1}, k_{2}$ are frequencies in the $x$ and $y$ directions, $\omega$ is the wave number of the propagating wave and $\epsilon$ is a phase constant. Here, Eq. (27) gives periodic trigonometric solution whereas Eq (28) gives the dark soliton solution.

The solutions shown as Eqs. (27) and (28) are those obtained by Yildrim [4].

One can try for solutions with $m=2,3$ and 4 which will become complicated. Attempts for solutions with $m \geq 5$ must be dropped out as algebraic equations with degrees greater than or equal to 5 are generally not solvable. 


\section{Conclusion}

In this paper of mine, the first integral method is successfully applied in finding exact solutions of Kundu-Mukherjee-Naskar equation that finds applications to the engineering of optical soliton propagation through optical fibers. It is inferred that the performance of this method is found to be effective and reliable. The method can be applied for finding exact solutions of many nonlinear evolution equations which frequently arise in the studies of social dynamics, science and engineering. One advantage of the method is that it is applicable to both integrable as well as non-integrable systems.

\section{References}

[1] Aliyu, A. I., Li, Y. and Baleanu, D. "Single and Combined Optical Solitons and Conservation Laws in (2+1)-Dimensions with Kundu- MukherjeeNaskar Equation", Chin. J. Phys., (2019). https://doi.org/10.1016/j.cjph.2019.11.001.

[2] Sulaiman, T. A. and Bulut, H. "The new extended rational SGEEM for construction of optical solitons to the (2+1)- dimensional Kundu-Mukhejee - Naskar model", Applied Mathematics and Nonlinear Sciences, 4(2), 513-522(2019). https://doi.org/10.2478/AMNS.2019.2.00048.

[3] Ekici, M., Sonmezoglu, A., Biswas, A. and Belic M. R. "Optical solitons in (2+1)-Dimensions with Kundu-Mukherjee-Naskar equa-tion by extended trial function scheme”, Chin. J. Phys. 57, 72 - 77 (2019). https://doi.org/10.1016/j.cjph.2018.12.011.

[4] Yildrim, Y. "Optical solitons to Kundu-Mukherjee-Naskar model with trial equation approach", Optik, 183, 1061 - 1065(2019). https://doi.org/10.1016/j.ijleo.2019.02.117.

[5] Singh, S., Mukherjee,A., Sakkaravarthi, K. and Murugesan,K.. "Higher dimensional localized and periodic wave dynamics in a new integrable (2+1)- dimensional Kundu-Mukherjee-Naskar-model", arXiv: 2001.06766v 1 [nlin. PS] 19 Jan 2020.

[6] Talarposhti, R. A., Jalili, P., Rezazadeh, H., Jalili, B., Ganji, D. D., Adel, W. and Bekir, A. " Optical soliton solutions to the (2 + 1)-dimensional Kundu-Mukherjee-Naskar equation”, Int. J. Mod. Phys. B, 2050102 (2020). https://doi.org/10.1142/S0217979220501027.

[7] F., Haas, Mahmood, S. "Nonlinear ion-acoustic solitons in a magnetized quantum plasma with arbitrary degeneracy of electrons, arXiv:1712.05339v 1 [physics. Plasm-ph] 14 Dec 2017. https://doi.org/10.1103/PhysRevE.94.033212.

[8] Guo, M., Fu, C., Zhang, Y, Liu, J. and Yang, H. " Study of Ion-Acoustic Solitary Waves in a Magnetized Plasma Using the Three-Dimensional Time- Space Fractional Schamel-KdV Equation”, Complexity, Volume 2018, Article ID 6852548, 17 pages https://doi.org/10.1155/2018/6852548.

[9] Feng, Z.S. "The first integral method to study the Burgers Korteweg-de Vries equation", J. Phys. A, 35 (2), 343 - 349 , (2002). https://doi.org/10.1088/0305-4470/35/2/312.

[10] Feng, Z. S. "On explicit exact solu-tions to the compound Burg ers- Korteweg-de Vries equation", Phys. Lett. A., Vol.293, 57 - 66 (2002). https://doi.org/10.1016/S0375-9601(01)00825-8.

[11] Feng, Z. S. "Travelling wave behavior for a generalized Fisher equation", Chaos, Soliton. Fract., Vol. 38, 481 - 488(2008). https://doi.org/10.1016/j.chaos.2006.11.031.

[12] Feng, Z. S. "Exact solution to an approximate sine- Gordon equa-tion in $(\mathrm{n}+1)$ - dimensional space", Phys. Lett. A, Vol.302, 64 - 76 (2002). https://doi.org/10.1016/S0375-9601(02)01114-3.

[13] Feng, Z. S. and Wang, X. H. “The first integral method to the two-dimensional Burgers- KdV equation”, Phys. Lett. A., Vol. 308,173 - 178 (2002). https://doi.org/10.1016/S0375-9601(03)00016-1.

[14] Feng, Z. S. and Knobel, R. "Travelling waves to a Burgers- Korteweg - de Vries equation with higher order nonlinearities", J. Math. Anal. Appl., Vol.328, No. 2, 1435 - 1450 (2007). https://doi.org/10.1016/j.jmaa.2006.05.085.

[15] Zhang, Z., Zhong, J., Dou, S. S., Liu, J., Peng, D. and Gao, T. "First Integral Method and Exact Solutions to Nonlinear Partial Differen-tial Equa tions arising in Mathematical Physics", Rom. Rep. Phys. 65(4), 1155-1169 (2013).

[16] Singh, S. S. "Soliton solutions of a generalized Klein-Gordon equation with power-law nonlinearity via the first integral method", International Journal of Mathematics and Physics 9, №2,116-121 (2018). https://doi.org/10.26577/ijmph.2018.v9i2.268. 